\title{
Actuation of Liquid Flow by Guided Acoustic Waves on Punched Steel Tapes with Protruding Loops
}

\author{
Alexander Backer ${ }^{*}$, Johannes Landskron, Klaus Stefan Drese, Gerhard Lindner \\ Institute of Sensor and Actuator Technology, Coburg University of Applied Sciences and Arts, 96450 Coburg, Germany
}

\begin{abstract}
In a biomimetic approach the feasibility of liquid flow actuation by vibrating protruding structures excited via guided acoustic waves is investigated. Inspired by periodically beating cilia the loop part of a punched metallic hook-and-loop tape with tilted protruding loops was used as a waveguide for plate waves in water. Such waves were excited in the frequency range of $110 \mathrm{~Hz}$ to $220 \mathrm{~Hz}$ by directly coupling the tape to a loudspeaker membrane. A flow generated in the tilt direction of the loops with velocities up to $60 \mathrm{~mm} \cdot \mathrm{s}^{-1}$ was visualized by ink droplets deposited on the tape. The phenomenon persisted, when the protruding length of the loops was reduced by decreasing the protrusion angle. However, after closing the punch holes near the loops with sticking tape streaming could not be observed any longer. The same happened with open punch holes when the ink was replaced by glycerol. Low-frequency acoustic streaming around vibrating sharp edges is proposed as an explanation for the observed phenomena. Applications are expected with respect to the modification of flow profiles and the enhancement of transport processes along and across liquid-solid boundaries.
\end{abstract}

Keywords: bio-inspired, guided acoustic waves, metallic hook-and-loop tape, artificial cilia, liquid propulsion

Copyright (C) The author(s) 2021.

\section{Introduction}

Liquid flow generation in narrow channels at small Reynolds numbers remains to be a challenging task in particular for microfluidic devices ${ }^{[1,2]}$. Nature, on the other hand, provides various concepts for biological flow generation ${ }^{[3]}$, among them motile cilia, which are particular effective for the actuation of directed liquid flow under such circumstances ${ }^{[4]}$. In the trachea, for instance, mucus is swept out of the airways by the oscillating motion of bundles of cilia forming the so-called mucociliary escalator of the respiratory epithelium ${ }^{[5,6]}$. This concept is characterized by the following features: Individual cilia are fingerlike soft-tissue structures extending from a basal cell membrane into a surrounding liquid. Directed flow is actuated by a non-reciprocal oscillatory motion of such cilia, consisting of an effective stroke exerting maximal momentum transfer to the liquid and a consecutive recovery stroke minimizing the drag force on the liquid by a corresponding change of their shape ${ }^{[7-10]}$. The periodic bending of motile cilia is driven by dynein molecular motors sliding internal microtubules along each other ${ }^{[11]}$. In many cases, such as in the mucociliary escalator, the motion of many closed packed individual cilia is synchronized forming metachronal waves thus enhancing the flow actuation $^{[4,12-15]}$.

Inspired by these attractive features numerous attempts to construct and to operate artificial cilia have been undertaken and various mechanisms for their actuation were introduced ${ }^{[16-18]}$. Among them were solutions operating by oscillating external electric fields ${ }^{[19-21]}$ or magnetic fields acting on incorporated magnetic nanoparticles ${ }^{[22-35]}$, by optical ${ }^{[36]}$ or pneumatic ${ }^{[37]}$ internal deformation of the ciliate body or by shaking the base-layer substrate ${ }^{[38-43]}$. Many of these attempts were focused on the reconstruction of the naturally occurring asymmetric bending trajectories of individual cilia by using flexible materials such as electroactive polymers or polymer nanorods containing iron oxide nanoparticles $^{[44]}$. Collective motions of arrays of artificial cilia resembling metachronal waves were achieved with rather sophisticated control systems ${ }^{[4-47]}$. In spite of these widespread research activities and the attractive features of such artificial cilia, however, their implementation in commercial products is rather rare up to now ${ }^{[48]}$.

\footnotetext{
*Corresponding author: Alexander Backer
}

E-mail: alexander.backer@hs-coburg.de 
In contrast to the bottom-up approach used in most of the studies mentioned above, i.e. starting with the construction of individual cilia with properties similar to natural archetypes, we looked for a commercially available object with certain structural similarities with ciliary assemblies and a simple means to excite asymmetric oscillations of the structures protruding into the liquid. As a result of this, we employed a punched metallic tape with skew protruding loops, which is a component of a loop-and-hook fastener, and guided acoustic waves excited by a loudspeaker coupled to one end of the tape for the excitation of a vibrational motion of the loops. This model system fulfilled at least some of the requirements of motile cilia arrays: An asymmetry of the trajectory of the tips of the loops is achieved by the retrograde elliptical deflection in the guided acoustic wave and their skew orientation. Moreover, resulting from the wave motion of the tape, the oscillations of the individual loops were synchronized with a fixed difference in phase resembling to a metachronal wave. On the other hand, however, there are substantial discrepancies to biological cilia, in particular due to the higher stiffness of the loops and the smaller differences between the forward and backward trajectories of the loops because of the small displacement amplitudes of the acoustic waves. Therefore, the feasibility of this approach was to be studied experimentally.

A similar approach for the actuation of cilia-like structures by periodic deformations of the base-layer was recently introduced by Rockenbach et al. ${ }^{[49]}$, who achieved flow velocities up to $0.3 \mathrm{~mm} \cdot \mathrm{s}^{-1}$ with flaps asymmetrically attached to an array of pneumatically deformable membranes. In our case the periodic deformation of the base-layer, i.e. the metallic tape, resulted from the propagation of acoustic waves guided along the interface between a solid surface and a liquid. Among them, Rayleigh waves and Lamb waves are featured by large displacement amplitudes at the surface of a corresponding waveguide and retrograde elliptical particle trajectories resulting from coupled longitudinal and transverse vibrations ${ }^{[50]}$. Even without protruding cilia such waves have proven their ability to generate liquid flow in different microfluidic applications ${ }^{[51-53]}$. The acoustic streaming phenomenon resulting from a vibrating flat solid surface in contact with the liquid, however, turned out to be rather ineffective at low frequencies, e.g. below $10 \mathrm{kHz}$. Therefore it was proposed to increase the propulsion effectivity in particular at low frequencies by the attachment of protruding cilia-like structures to the vibrating surface, whose motion follows non-reciprocal trajectories as well ${ }^{[54]}$. A dynamic deformation of these structures as known from biological cilia would certainly be advantageous to enhance the propulsion effect, but seems to be not necessary for an asymmetry of the resulting momentum transfer to the liquid and thus the generation of a directed flow. On thin plates such as the metallic tape, fundamental modes of Lamb waves can easily be excited at low frequencies by remote electrodynamic transducers, in particular the dispersive antisymmetric fundamental mode $\mathrm{A}_{0}$, which converts into a Quasi-Scholte Plate (QSP) mode, when the plate is immersed into a liquid ${ }^{[55-57]}$. At very low frequencies they are also named flexural waves due to their flexing motion.

In the following sections we report on the results of experiments with the vibrating punched metallic tape with protruding loops immersed into a water basin. This includes the demonstration of the generation of a directed flow at certain excitation frequencies and investigations of the influence of parameters such as the spatial orientation of the protruded loops and the viscosity of the liquid and of the importance of the punched holes neighbouring the protruding loops. For an explanation of the observed phenomena a model of low-frequency acoustic streaming around vibrating sharp edges as proposed by Ovchinnikov et al. ${ }^{[58]}$ has been employed for numerical calculations, whose preliminary results are presented as well. The observed phenomena already indicate a remarkable potential for future applications of flow management with remote power actuators.

\section{Acoustic background}

A fundamental requirement for a fluid propulsion excited by a vibrating plate is a movement out of the unperturbed surface of the plate. In case of an infinite plate such oscillations are described by Lamb waves. These waves occur in antisymmetric (A) and symmetric (S) form. The lowest order modes $\left(\mathrm{A}_{0}, \mathrm{~S}_{0}\right)$ exist in the whole frequency space. However, the $\mathrm{A}_{0}$ Lamb mode exhibits larger sagittal displacement amplitudes at the 
surface than the $S_{0}$ mode ${ }^{[50]}$. Moreover, it is preferentially excited by an asymmetrically acting transducer and it converts easily into a QSP (flexing) mode when the waveguide is immersed into a liquid in a dipstick configuration $^{[56,57]}$. For these reasons the antisymmetric $\mathrm{A}_{0}$ Lamb mode is particular suited for the approach chosen in this paper.

The possibility of the transformation of the $A_{0}$ to the QSP mode is evidenced by the dispersion diagram of Lamb waves on a thin steel tape as calculated with the software tools Disperse (Version 2.0.20a) and MathWorks MATLAB (Fig. 1). It displays the coincidence of the $\mathrm{A}_{0}$ and the flexural wave mode at low frequencies, the similarity of the QSP and $\mathrm{A}_{0}$ phase velocity dispersion relations and in particular the strong dispersion, which limits the wavelength $\lambda$ to reasonably small values of a few centimetres when the frequency becomes small (e.g. $\lambda=6 \mathrm{~cm}$ at $200 \mathrm{~Hz}$ ). Small frequencies below $1 \mathrm{kHz}$ are preferred for the actuation of the punched metallic tape in order to avoid acoustic streaming effects from the deflections of the tape and to keep the excitation frequency below eigenfrequencies of the protruding loops and to achieve large deflection amplitudes of the metallic tape and movement of the loops in-phase with the vibration of the tape.

From structural acoustics flexural plate waves are known to be responsible for sound transmission in plates in that frequency range; at low frequencies, however, their dispersion relation coincides with that of $\mathrm{A}_{0} \mathrm{Lamb}$ modes ${ }^{[59]}$. This is corroborated by the dispersion calculations for a homogeneous plate, which ended up with identical results for both approaches in the frequency range below $300 \mathrm{~Hz}$ (Fig. 1). Therefore it is sufficient to consider only the $\mathrm{A}_{0}$ Lamb mode for the wave propagation on the steel plate in air outside the basin and the QSP wave within the liquid. Although the punched metallic tape can be considered as a perforated plate, whose dispersion properties may differ from that of an infinite homogeneous plate ${ }^{[60]}$, we assumed that the influence of perforation on the movement of the tape is rather small at the low frequencies chosen in this study. This assumption has been corroborated by dispersion calculations for a plate with a similar perforation pattern as the metallic tape; corresponding results are presented in section 4. In analogy, we continue to use the terminology
" $\mathrm{A}_{0}$ mode" and "QSP mode" for the guided acoustic waves on the perforated tape in the following, despite the fact, that the symmetry and boundary conditions are no longer exactly fulfilled.

\section{Materials and methods}

\subsection{Test devices}

A metallic hook-and-loop tape ("Metaklett", manufactured by Hölzel Stanz- und Feinwerktechnik GmbH \& Co. KG, Wildberg, Germany) was chosen as a suitable candidate for the tests of the concept. The loop part consists of a $0.25 \mathrm{~mm}$ thick and $w=30 \mathrm{~mm}$ wide punched steel tape, which bears on one side parallel rows of protruding loops produced by punching at a distance of $d=3.5 \mathrm{~mm}$ (Fig. 2a). These loops form a triple cascade and protrude from the tape plane at protrusion angles $\varphi$ of about $27^{\circ}$ to $45^{\circ}$ in the lower part which increases continuously with growing distance from the tape plane, i.e. their orientation is asymmetric in space (Figs. $2 b$ to $2 d$ ). Correspondingly their tip follows a non-reciprocal trajectory when an $\mathrm{A}_{0}$ Lamb wave propagates along the tape due to the elliptical motion of the basepoint of the loop on the tape. Within the liquid the elliptical basepoint motion of the tape will remain almost unchanged since the mode shape of the QSP is similar to that of the $\mathrm{A}_{0}$ mode. The drag force of the liquid tends to change the angle of attack of the loop in different ways depending on the direction of the relative motion between the loop and the liquid: This angle is increased, when the drag force of the liquid tries to lift the loop, whereas it is decreased by a drag force in opposite direction. Thus the effective area of the loop,

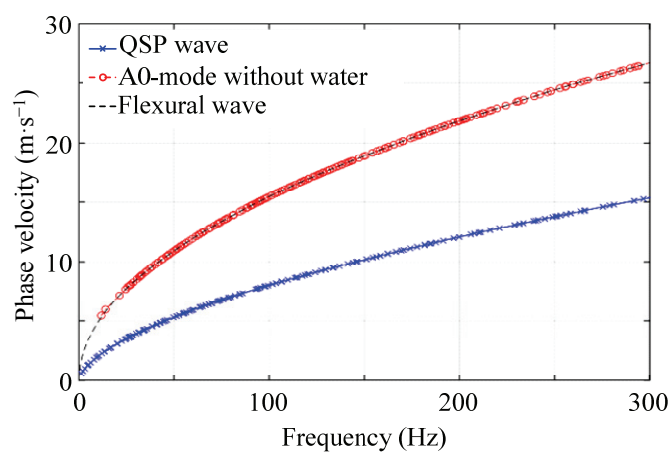

Fig. 1 Calculated phase velocities of the $A_{0}$ Lamb wave, the flexural plate wave and the QSP wave of a $0.25 \mathrm{~mm}$ thick steel tape as a function of frequency. 


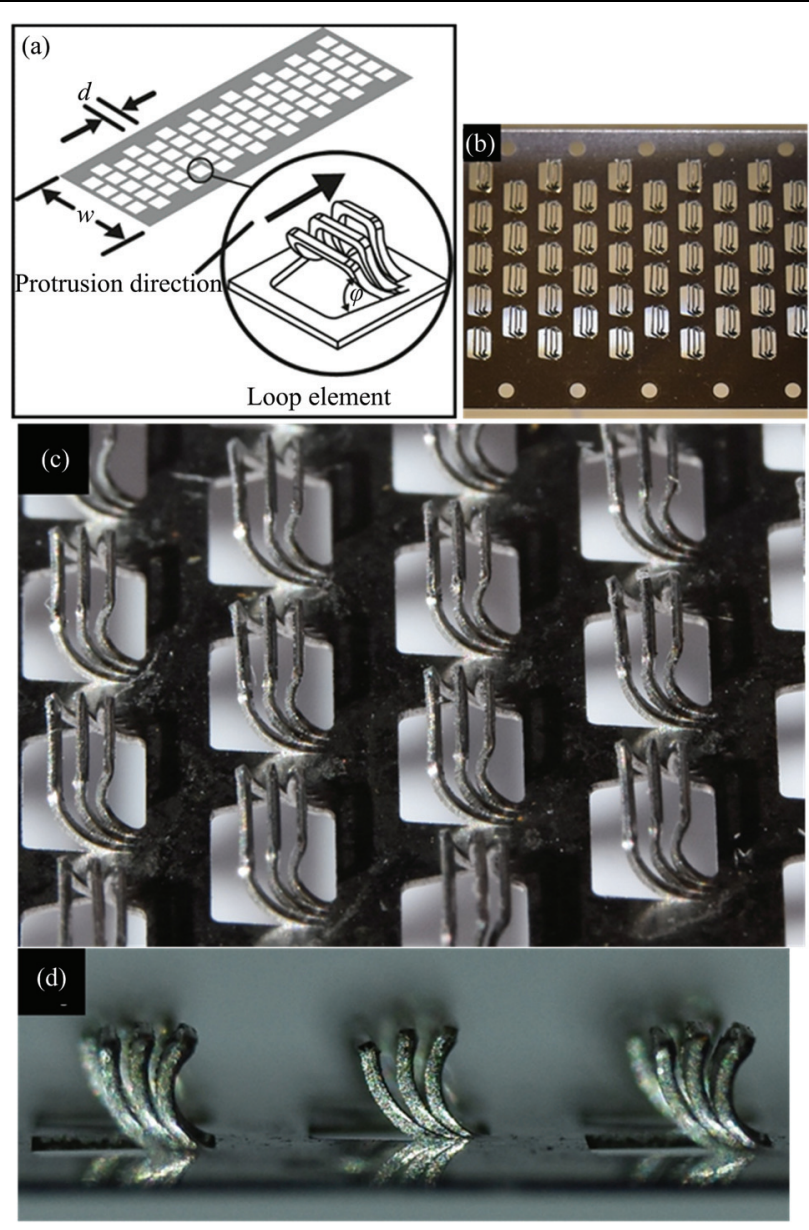

Fig. 2 Loop part of the metallic hook-and-loop tape "Metaklett" $(w=30 \mathrm{~mm}, d=3.5 \mathrm{~mm}$, protrusion angle $\varphi$ in the lower parts of the loops between $27^{\circ}$ and $45^{\circ}$ ). (a) Schematic drawing; (b) top view; (c) skew view; (d) side view.

which protrudes into the liquid, changes during a period of the elliptical motion resulting in an imbalance between a power and a recovery stroke, which is necessary for liquid propulsion. Moreover, since the loops were produced by a cold sheet metal forming process, which causes residual stress in the material, the bending stiffness of the loops presumably differs slightly between uplifting and bearing down.

\subsection{Experimental arrangement}

The experimental set-up consist of an open water basin, into which the long tape is partially inserted almost horizontally and held below the water surface by a fastener at one end, whereas the other end is connected with the membrane of a loudspeaker (Type (VW) $1 \mathrm{KM}$ $035454 \mathrm{~B}$ ) above the water surface (Fig. 3). This connection is formed in a manner that $\mathrm{A}_{0}$ Lamb waves are predominantly excited in the tape by the vibrating membrane of the loudspeaker, which propagate along the tape including the part immersed into the water. In this part the tape extends almost horizontally in order to exclude gravity effects on a streaming parallel to its surface. There are four different possibilities of the orientation of the tape - loops protruding upwards or downwards and bending of the loops towards the loudspeaker or away from it.

In the experiments the loudspeaker was driven via an amplifier (DYNACORD PAA880, Dynacord GmbH, Straubing, Germany) with sine signals from a waveform generator (Agilent 33521 A, Agilent, San Jose, USA) in the frequency range $100 \mathrm{~Hz}$ to $220 \mathrm{~Hz}$ and peak-to-peak voltages between $1 \mathrm{~V}$ and $9.5 \mathrm{~V}$. This frequency range was chosen because in pretests only there flow actuation could be detected. The streaming was visualized by blue ink droplets, which were deposited on the tape surface by a syringe. Videos of the behaviour of the ink were recorded with a digital camera (Nikon D7200) with a frame rate of 29.97 frames per second (Figs. 4, 8 and 9, videos $2,3,4,5,6,7$ and 8 in the supplementary materials); for the videos for the determination of the frequency dependence of the streaming velocity (Fig. 10) another digital camera (Sony Alpha 6300) with a frame rate of $50 \mathrm{fps}$ was used. The wave motion on the water-covered tape was measured by a laser Doppler vibrometer (PSV 400M, Polytec GmbH, Waldbronn, Germany), which scanned the tape surface from a position above the water surface with vertically downwards oriented laser beams. The detailed records of the streaming around a single punch hole were obtained by a high-speed camera (KEYENCE, VW-600C, $1000 \mathrm{fps,}$ videos 10 and 11 in the supplementary materials) and a digital camera (Sony Alpha 6300, 100 fps, Figs. 11 and 12 , videos 9 and 12 in the supplementary materials).

\section{Experimental results}

\subsection{Preliminary experiments with a smooth steel tape}

In preliminary experiments, a smooth steel tape with a thickness of $0.2 \mathrm{~mm}$ and width of $30 \mathrm{~mm}$ without punch holes was connected to the loudspeaker and inserted into the water tank in order to check whether waves propagating along a plate without protruding structures would be able to excite any streaming. Sound waves with frequencies between $100 \mathrm{~Hz}$ and $180 \mathrm{~Hz}$ were excited on this plate and ink droplets were 


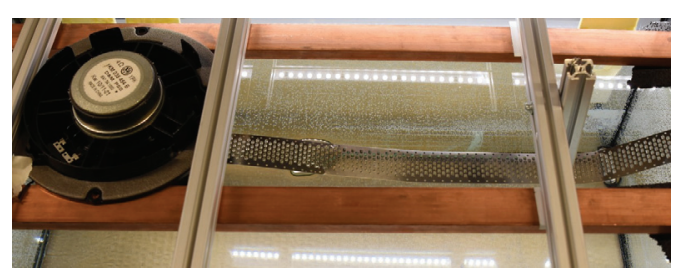

Fig. 3 Experimental set-up showing the water basin, the loudspeaker and the horizontally arranged metallic tape.
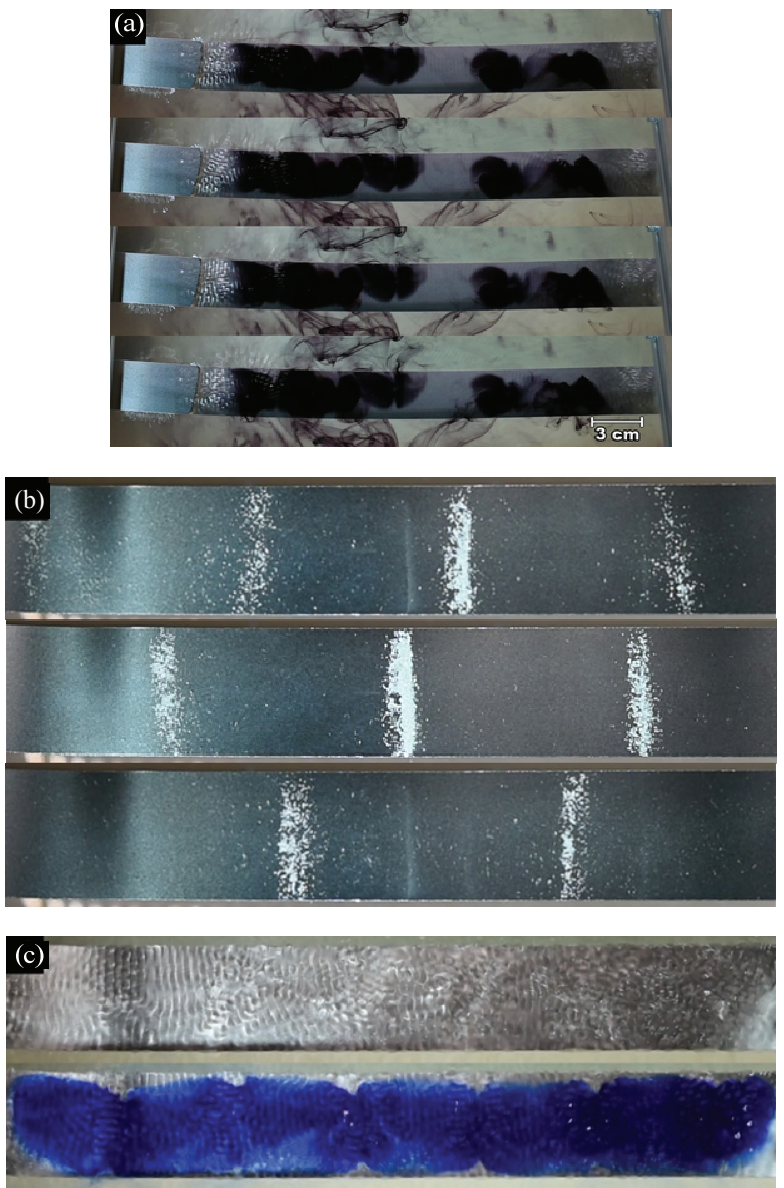

Fig. 4 (a) Immersed smooth steel tape continuously excited by the loudspeaker. Resonance vibrations were at $135 \mathrm{~Hz}$, time step $2 \mathrm{~s}$ between each snapshot. No directed flow of ink was observable. (b) Vibrational resonances observed in air at frequencies of $225 \mathrm{~Hz}$ (upper), $175 \mathrm{~Hz}$ (middle) and $120 \mathrm{~Hz}$ (lower). (c) Loading of the immersed vibrating plate vibrating at a resonance frequency of $150 \mathrm{~Hz}$ in water without (upper) and with a glycerol layer tinted with blue ink (lower).

deposited on the plate in order to visualize any flow. Vibrational resonances were recorded at distinct frequencies of $105 \mathrm{~Hz}, 135 \mathrm{~Hz}$ and $165 \mathrm{~Hz}$ indicated by strong stirring of the ink and standing waves on the water surface, but no directed flow of the ink was observed (Fig. 4a). The existence of distinct vibrational resonances was proven by the accumulation of quartz sand in the nodes of standing waves without water loading as shown by Fig. 4b. In addition, the deposition of glycerol on the plate obviously did not alter the resonance effects observed at the immersed plate in the water basin (Fig. 4c).

\subsection{Numerical calculations of the wave propagation on punched tapes}

In order to check whether the replacement of the steel plate by the metallic tape with the loops would change the propagation of the acoustic waves significantly, both dispersion calculations for a model structure containing perforations and loops as well as Scanning Laser Doppler Vibrometer (SLDV) records of the tape vibrationally excited by the loudspeaker were performed. For the numerical calculations, a model structure was established consisting of a single unit cell formed by a basic plate with a perforation and a simple loop according to Fig. 5a. The chosen dimensions of the basic plate with a length $a=3.5 \mathrm{~mm}$ and a thickness $0.2 \mathrm{~mm}$ and of the loop with a side length of $2.5 \mathrm{~mm}$, a joint width of $0.5 \mathrm{~mm}$ and a protruding angle of $30^{\circ}$ corresponded to those of the hook-and-loop tape used in the experiments. Material parameters typical for a steel tape (density of $7850 \mathrm{~kg} \cdot \mathrm{m}^{-3}$, Young's modulus of $200 \mathrm{GPa}$ and Poisson's ratio of 0.3 ) were chosen and the dispersion diagrams and the mode shapes of the $\mathrm{A}_{0}$ mode were calculated with the structural mechanics module of the software tool COMSOL Multiphysics 5.4, using Floquet periodic boundary conditions to account for the

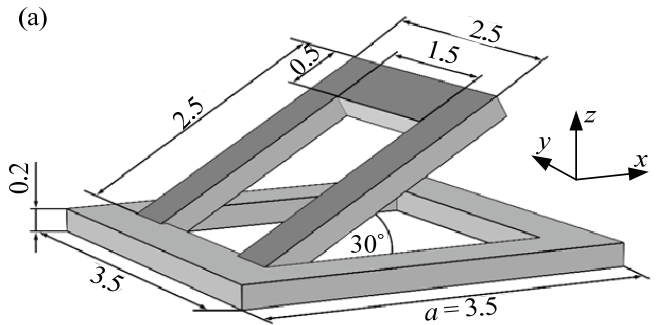

(b)

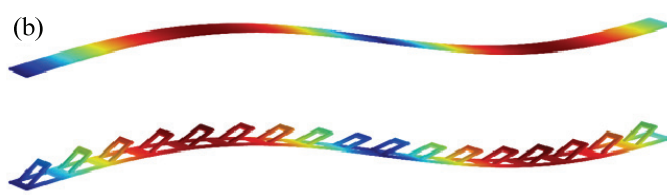

Fig. 5 (a) Geometrical model of the unit cell used for the numerical calculation of the dispersion diagram. All measures were given in $\mathrm{mm}$. (b) Simulation result showing the mode shape of the lowest order antisymmetric Lamb mode of the metallic tape without and with punch holes and protruding loop structures. 


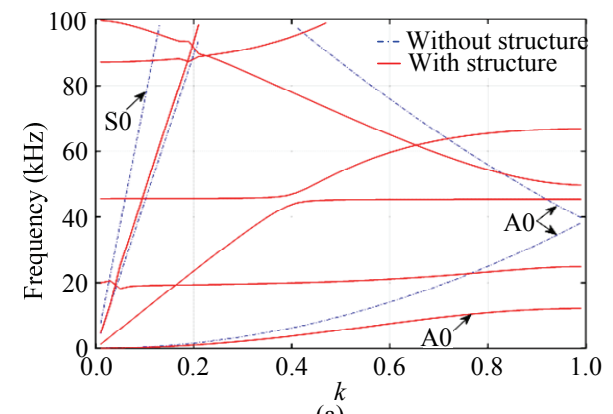

(a)

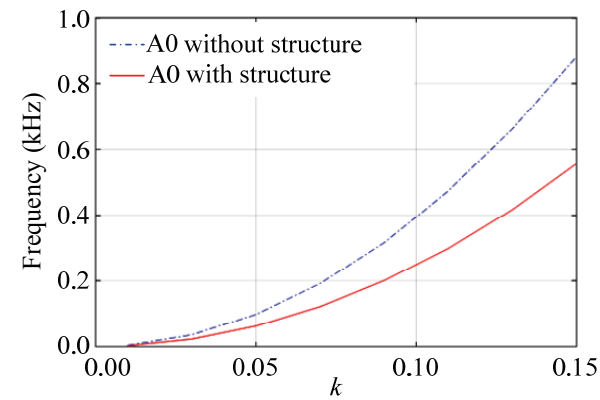

(b)

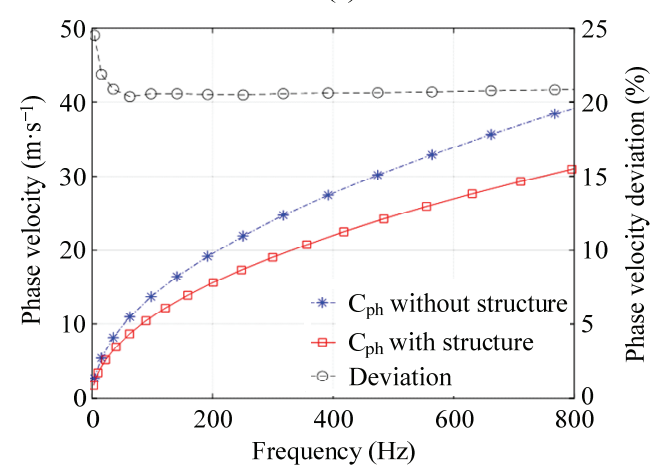

(c)

Fig. 6 (a) Dispersion diagrams for the unstructured case (blue, dash dot) and structured case (red, line); (b) enlarged section of the dispersion diagram for $k<0.15$; (c) frequency dependence of the phase velocities comparing the unstructured case (blue, stars) with the structured one (red, squares) and the relative difference (black, circles).

periodicity. A calculated mode shape of an $\mathrm{A}_{0}$ mode on such a model tape excited with the same wave number is shown in Fig. 5b. Since the wave propagation is assumed to be unidirectional into the direction of the long side of the basic plate ( $x$ direction), it is sufficient to consider the $\Gamma$-X-part of the first Brillouin zone with a corresponding wave number $k_{x}=\pi k / a$, where $k$ is swept from 0 to 1 in the solving process. As a result of plotting the calculated eigenfrequencies against the parameter $k$, the dispersion diagram of Fig. 6a exhibiting the existing modes in the frequency range between $0 \mathrm{kHz}$ and $100 \mathrm{kHz}$ is obtained. Included in this figure is the dispersion diagram of the unstructured basic plate, which contains a symmetric and an antisymmetric Lamb wave. There are substantial differences between both dispersion diagrams, including band gaps know from phononic crystals in the diagram of the perforated plate. But, at the small frequencies below $1 \mathrm{kHz}$ used throughout this paper, only the $\mathrm{A}_{0}$ modes are excited (Fig. 6b), for which the differences are negligible. In our case the $k$ values are near $k=0.12$, where the influence of the additional structure is still rather weak. This is corroborated by the dispersion relation of Fig. 6c for the low frequency region, where the modification of the propagation velocity due to the additional structure is in the order of $20 \%$, whereas the shape of the dispersion curve remains similar and shows a nearly constant deviation of about $20 \%$, i.e. a small adjustment of the plate thickness would lead to the exact same dispersion diagram in the frequency range of interest. Therefore the interpretation of the vibrational motion of the tape in terms of an $\mathrm{A}_{0}$ mode in air or a QSP in water remains justified. This interpretation is also corroborated by the SLDV records (Fig. 7). The corresponding vertical displacement velocity pattern at $185 \mathrm{~Hz}$ excitation frequency clearly reveals a single mode of a plane wave with a wavelength of $55 \mathrm{~mm}$ and thus a phase velocity of $10.2 \mathrm{~m} \cdot \mathrm{s}^{-1}$ propagating along the whole length of the tape without measurable attenuation, which was assigned to the QSP mode. These measured values agree reasonably with the calculated QSP dispersion curve from Fig. 1.

\subsection{Experiments with a vibrating metallic hook- and-loop tape}

In the following experiments the behaviour of ink and glycerol droplets on the metal tape was recorded with the video camera for different orientations of the tape, where the waves were always excited by the loudspeaker on the left side, i.e. the waves propagated from the left to the right. At first, the tape was arranged with the loops standing upwards and suspended at the left edges of the punched holes towards the loudspeaker, i.e. tilting to the right. With a peak-to-peak actuation voltage of $5 \mathrm{~V}_{\mathrm{pp}}$ at $180 \mathrm{~Hz}$ a streaming of the ink to the right was observed (Fig. 8). When the orientation of the tape was turned into the opposite direction, i.e. the loops were suspended at the right edges of the punched holes and tilting to the left, the streaming of the ink was 


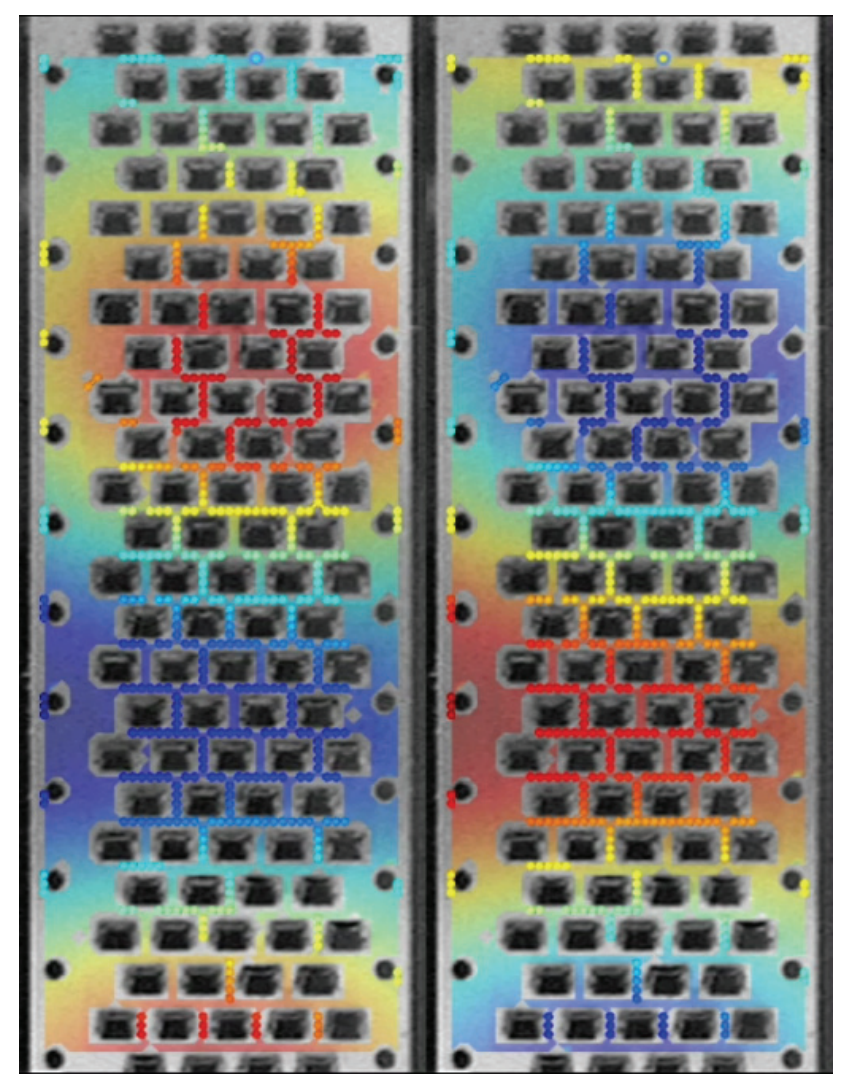

Fig. 7 Two subsequent SLDV records of wave propagation on punched metallic tape with a time difference of $2.7 \mathrm{~ms}$.

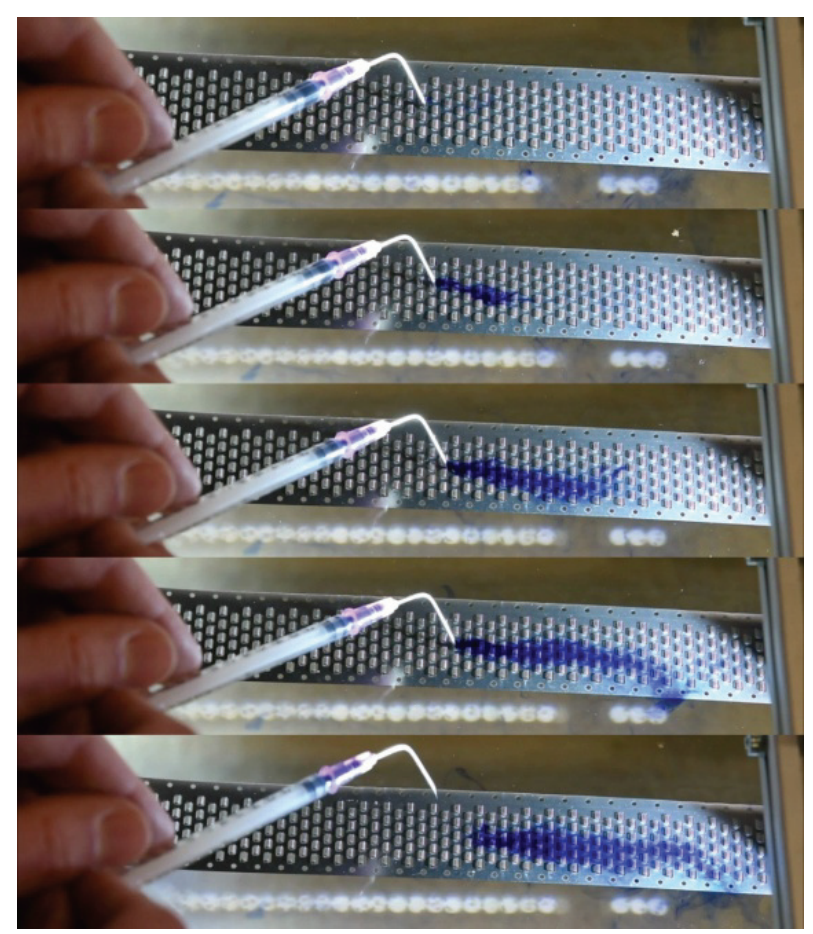

Fig. 8 Ink flow observed at an excitation frequency of $180 \mathrm{~Hz}$ over loops tilting to the right showing a flow to the right. Snapshots were taken with time step of $2 \mathrm{~s}$.

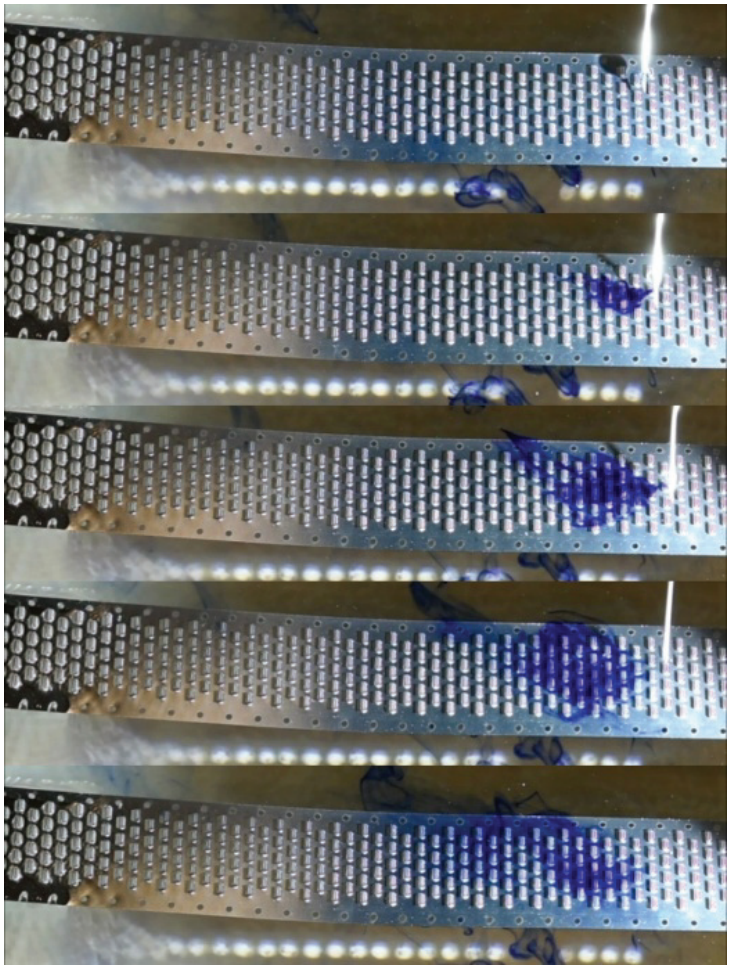

Fig. 9 Ink flow observed at an excitation frequency of $180 \mathrm{~Hz}$ over loops tilting to the left showing a flow to the left. Snapshots were taken with time step of $2 \mathrm{~s}$.

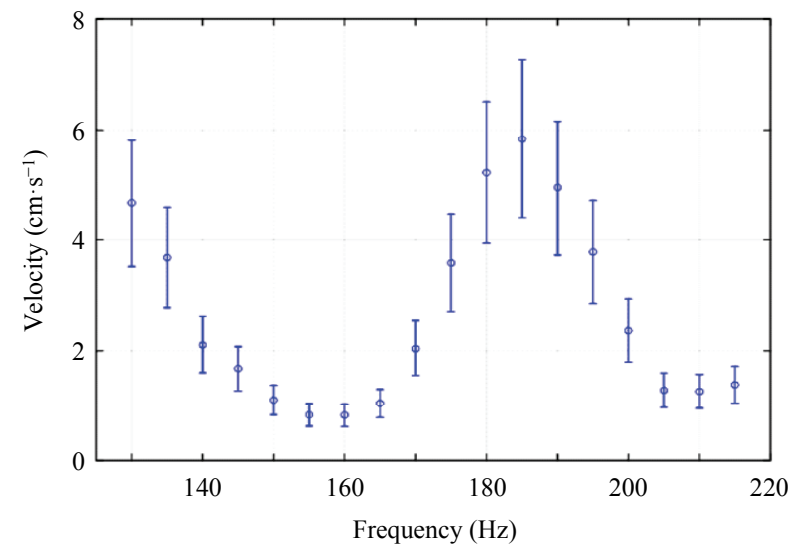

Fig. 10 Frequency dependence of ink flow velocity obtained with a tape with knocked down loops.

directed to the left with the same actuation conditions (Fig. 9).

Turning the tape upside down with the loops now standing downwards, no ink flow was achieved with ink droplets deposited on the smooth side of the tape. Obviously, the loops were responsible for the flow actuation and the flow direction is determined by the tilt orientation. Compared to plane plates the symmetry of top down is broken by the existence of the loops. 
In order to check the importance of the length and the spatial orientation of the loops, they were knocked down leaving back only short snags, but still almost the same ink flow was observed, i.e. a flow to the left, when the snags were suspended at the right edges and tilted to the left. The small protrusion length of the snags seems to be sufficient for flow actuation and determination of the flow direction. On the other hand, replacing ink by glycerol, no flow of glycerol in contrast to persisting flow of ink was observed, which outlines the importance of the viscosity for the flow phenomenon.

Finally, after closing a part of the holes with sticking tape, no ink flow could be induced in these sections of the tape, but in the remaining part of the tape with open holes the ink flow emerged with about the same velocity and direction as on the uncovered tape.

The actuation of directed flow of ink was observed all across the whole area of the tape with open holes, which allowed a quantitative determination of the flow velocity from the video records (Fig. 10). The error bars resulted from the evaluation of the progression of the ink front at several measurement sites. Significant flow occurred over a wide range of frequencies between $130 \mathrm{~Hz}$ and $215 \mathrm{~Hz}$ with a maximum of $60 \mathrm{~mm} \cdot \mathrm{s}^{-1}$ at $185 \mathrm{~Hz}$.

The importance of the shape of the protruded loops, in particular of the joints, was checked with observations from tape portions where the joints of the loops were removed in comparison with observations from tape portions with unchanged loops. Without joints the propulsion effect was much weaker as for the case with joints. These results were derived from corresponding video records (videos in supplementary file).

Summarizing, a directed flow of ink near the tape surface caused by QSP was only observed on the side of the metallic tape where the loops were protruding beneath punched holes. No flow was found on the smooth backside or when holes were closed by sticking tape. The direction of streaming is determined by the suspension of the protruded loops; their length, however, is of minor importance with respect to the flow velocity achieved. The flow actuation is restricted to low-viscosity liquids; with glycerol, whose viscosity is about 1000 times larger than that of water, no flow could be generated. The streaming velocity varied between
$10 \mathrm{~mm} \cdot \mathrm{s}^{-1}$ and $60 \mathrm{~mm} \cdot \mathrm{s}^{-1}$ depending on frequency with a distinct maximum at $185 \mathrm{~Hz}$.

\section{Discussion}

From the experimental results it can be concluded that the geometrical structure of the hook-and-loop tape and its movement in the liquid are the main reason for flow generation. Acoustic streaming known from guided acoustic waves along plane structures can be excluded, since at the vibrating unpunched plate no flow could be excited. This indicates at the first glimpse that the bio-inspired similarity of the geometrical structure and motion of the vibrating hook-and-loop tape with motile cilia arrays is a crucial factor for the observed effects. A conclusive explanation of the experimental findings, however, demands for a more detailed consideration of the interaction of the vibrating structures with the liquid.

In particular, the following phenomena have to be explained by a suitable mechanism:

(a) There is no flow when the holes beneath the protruding loops are closed with tape.

(b) The direction of the flow is always oriented in the tilt direction of the loops.

(c) Flow is only observed with a low-viscosity liquid such as water, not with a high-viscosity liquid such as glycerol.

(d) The flow persists when the loops were knocked down, i.e. when the protrusion angle is reduced.

(e) The flow is strongly suppressed when the joint of the loops are removed.

To this end, the fluid dynamics resulting from the interaction of the vibrating structures with the liquid is analysed and recorded with additional video sequences with larger magnification as before and numerical simulations are performed based on a theoretical approach developed by Ovchinnikov et al., which explains the acoustic streaming effect around sharp edges ${ }^{[58]}$.

\subsection{Fluid dynamic considerations}

At first, the symmetry of the structure has to be considered: Both the top-down and the rightleft-symmetries are broken due to the skew protruding loops, which are fixed on one side of the punch holes and which protrude to the upper side. Therefore, even a purely vertical movement of the tape without any hori- 
zontal components may cause a horizontal component of the liquid movement because of the asymmetry of the structure.

Next, essential parts of the structure needed for flow actuation have to be identified: According to (a) and (e) the joints of the loops neighbouring holes seems to be responsible. They follow the cyclic deflections of the tape due to the QSP waves, i.e. they move periodically on almost vertical trajectories in the liquid with changing velocities determined by the excitation frequency and the deflection amplitudes. With a peak velocity of $v_{p}=78 \mathrm{~mm} \cdot \mathrm{s}^{-1}$ obtained from LDV measurements with a signal amplitude from the waveform generator of $9.5 \mathrm{~V}$, a characteristic diameter $l=0.25 \mathrm{~mm}$ of the joints of the loops and a kinematic viscosity $v=1 \mathrm{~mm}^{2} \cdot \mathrm{s}^{-1}$ of water, we obtain with:

$$
R e=\frac{v_{p} \cdot l}{v},
$$

a Reynolds number of $R e=20$ in water, which is representative for symmetric laminar streaming around the loops and vortex formation behind the loops and which is near to the vortex shedding regime ${ }^{[61]}$. At this order of magnitude of the Reynolds number, however, the time inversion symmetry is broken, which is a necessary requirement for the occurrence of acoustic streaming. For glycerol with $v=1170 \mathrm{~mm}^{2} \cdot \mathrm{s}^{-1}$, on the other hand, a Reynolds number of $R e=0.02$ is obtained, which is deep in the laminar flow regime. Therefore, vortex generation by the vibrating joints of the loops seems to be mandatory for the flow actuation ${ }^{[20]}$, which explains observation (c).

In contrast to the joints of the loops, the yokes of the loops seem to be of minor importance for flow actuation. For artificial cilia the pumping efficiency can be expressed by the sperm number $S p$, which characterizes the ratio of Stokes drag forces to bending forces and is defined by:

$$
S p=L \cdot\left(\frac{4 \pi \mu \omega}{E \cdot I}\right)^{\frac{1}{4}},
$$

where $L$ length of slender cylinders, $\mu$ viscosity, $\omega=2 \pi f$ angular frequency, $E$ Young's modulus, $I=b h^{3} / 12$ area moment of inertia ${ }^{[43]}$. With typical values for the metallic yokes of the loops, i.e. $L=2.5 \mathrm{~mm}, \mu=10^{-3} \mathrm{~Pa} \cdot \mathrm{s}$, $f=185 \mathrm{~Hz}, E=200 \mathrm{GPa}, b=1 \mathrm{~mm}, h=0.25 \mathrm{~mm}$, a value of $S p=0.04$ is obtained, which is much smaller than the value of $S p=3$ identified for optimal pumping performance of artificial cilia ${ }^{[62]}$. That means that the yokes are rather stiff and that the joints closely follow the baseline vibration of the plate surfaces. Therefore the flow actuation mechanism differs substantially from liquid propulsion with flexible flagellas ${ }^{[9,63]}$.

\subsection{Explanation by low-frequency acoustic stream- ing around sharp edges}

In order to gain inside in the flow actuation mechanism video sequences of the spread of ink are recorded from which snapshots are shown in Fig. 11. They evidence the development of an extended vortex at the left edge of the punched hole, which becomes asymmetric towards the right edge where the joints of the loops are located. In addition they resemble a second jet-like streaming to the right originating from the vibrating joints. Both the original vortex-like streaming and the jet-like streaming form a streaming to the right, which extends over several rows of punch holes (Fig. 11). This formation of a directed streaming only happens on the upper side of the tape, where the loops are protruding upwards, in contrast to the lower side, where no directed flow could be observed. With a high-speed camera this development of a jet-like streaming originating from the vibrating joints in addition to the dominating vortex was also recorded with higher temporal resolution (see videos 10 and 11 in the supplementary materials). Considering the regular arrangement of rows of punch holes, it becomes obvious that this directed jet-like streaming is successively supported by the individual streaming contributions from each row (Fig. 12), which explains the long-range extension of the streaming phenomenon across the tape.

Jet-like streaming caused by a vibrating body with a sharp edge in a liquid has recently been described by Ovchinnikov et al. ${ }^{[58]}$. They observed a strong outflow of fluid along the centre line of a sharp edge and an inflow from the sides and they provided numerical calculations using a perturbation theory approach for solving the Navier-Stokes equation. Similar observations of acoustic streaming around vibrating sharp edges were recently reported by several authors, in 


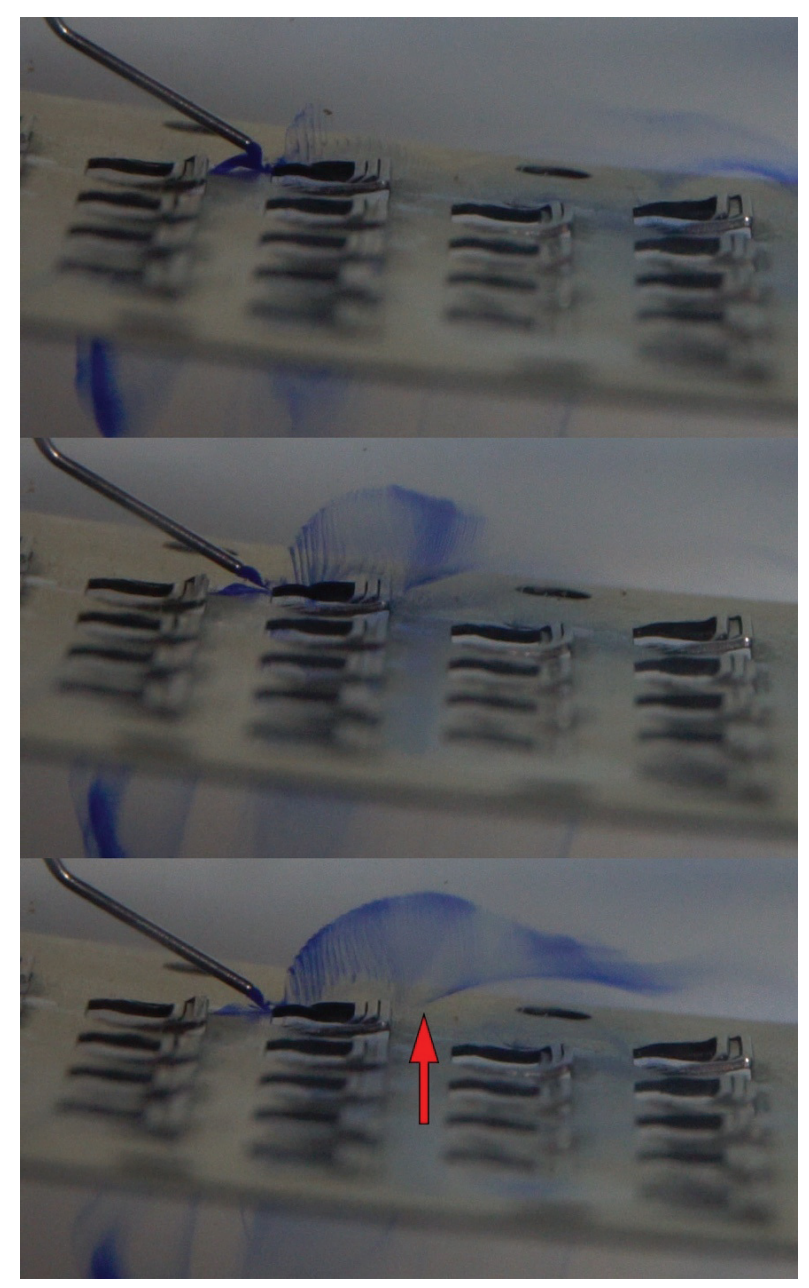

Fig. 11 Formation of a vortex above a vibrating punch hole and of a jet stream (arrow) from the protruded joints in the tilt direction of the joints. The sequence of ink fronts from the periodic pushing by the vibrating structure is visible in the large vortex. There is no horizontal streaming effect of the ink below the tape. Snapshots were taken with time steps of $30 \mathrm{~ms}$.

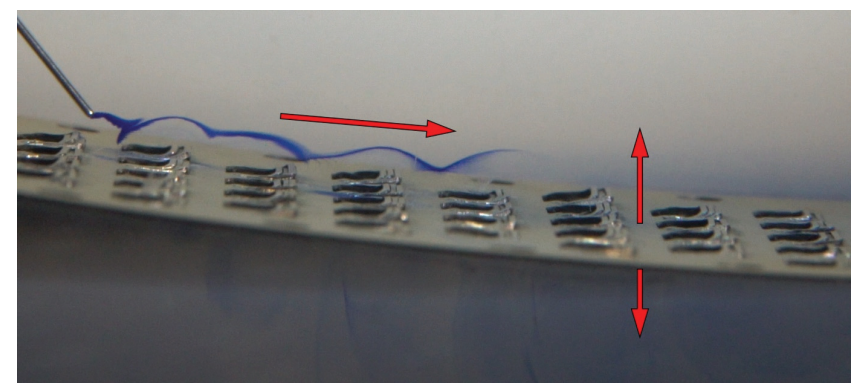

Fig. 12 Visualization of a far-reaching ink flow over several rows of protruding loops when the ink is introduced without contact to the tape surface.

particular with respect to micromixing in microfluidic

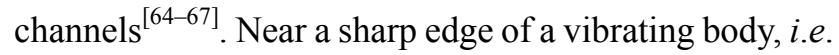
when the radius of curvature of the edge is much smaller than the width $\delta$ of the viscous boundary layer with $\delta=\sqrt{\frac{v}{2 \pi f}}$ according to Ovchinnikov et al. ${ }^{[58]}$, a novel type of acoustic streaming occurs which drives the jet. In our experiment, the width of the viscous boundary layer was $\delta=29 \mu \mathrm{m}$ at a frequency of $185 \mathrm{~Hz}$. The radius of the edges of the loops was determined to be in the range of $10 \mu \mathrm{m}-25 \mu \mathrm{m}$ by a digital microscope (KEYENCE VHX-7000) and thus is smaller than the $29 \mu \mathrm{m}$ boundary layer width calculated above. Therefore we conclude that the acoustic streaming mechanism described by Ovchinnikov et al. is responsible for the flow effects observed with the vibrating punched tape. All the empirical findings (a) to (e) listed above can be explained with this model, including the vanishing of the flow effect in glycerol, the importance of the punch holes for closing the vortices, the persistence of the flow when the loops were knocked down, i.e. the small influence of the protrusion angle and the increase of the flow velocity with higher oscillation amplitudes near vibrational resonances as suggested by Fig. $10^{[58]}$. In addition, the synchronized oscillations of neighbouring rows of loops and holes explain the observed far-reaching transport of the ink (Fig. 12), since it will be supported by the sequence of jet flows originating from each of the vibrating joints. Correspondingly, numerical calculations following their perturbation theory approach with an idealized geometry of a punched hole and loop configuration resulted in strong flow effects around the edges of the hole and in particular the joint of the loop (Fig. 13). The streamlines nicely replicate the flow phenomena observed in the experiments.

\subsection{Summarizing evaluation}

As a summary, the flow generation mechanism turns out to be quite different from that of motile cilia. Acoustic streaming caused by vibrating sharp edges in a viscous boundary layer together with inertial effects of the liquid were identified as key factors. Nevertheless, essential features of the natural archetype such as periodic vibrations of an array of protruding structures near a boundary and the asymmetry of the drag force exerted on the liquid are also incorporated in our artificial device. Another similarity with natural cilia arrays is suggested by the shape of the flow field actuated in the overlaying liquid: The generation of strong tangential flow has both 


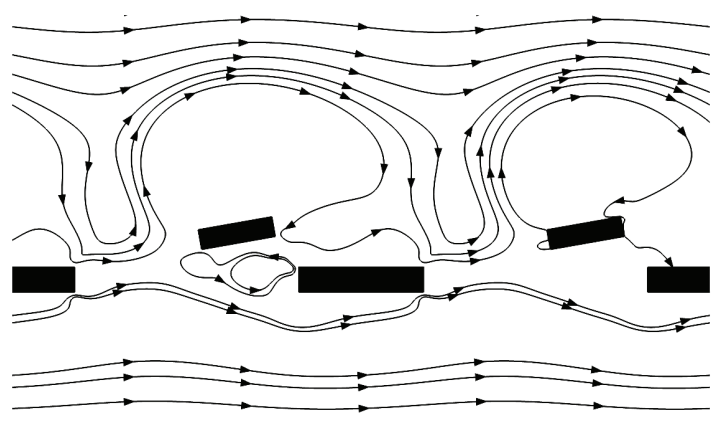

Fig. 13 Streamlines showing the flow calculated by CFD over a simplified 2D model of the oscillating hook-and-loop tape.

been observed at swimming Paramecium, a single-cell eukaryote ${ }^{[68]}$, and with our vibrating tape device (see e.g. Fig. 12). Therefore in a broader sense it seems justified to consider it as bio-inspired as well. But the regime of operation is quite different, i.e. this system will neither be able to replace motile cilia in their natural applications nor other types of artificial cilia developed for such operative ranges. On the other hand, this concept may allow a novel access to other tasks, in which liquid flow generation near boundaries is desired, e.g. for the equalization of concentration gradients near electrodes in electrochemical systems, for the removal of gas bubbles in electrolytic cells or in microfluidics at larger Reynolds numbers.

Compared to other types of artificial cilia the vibrational excitation via guided acoustic waves is rather simple and effective; external installations such as distant electromagnets or pneumatic installations are not necessary. The loudspeaker used in our experiments may become replaced in future by other types of vibration generators such as small electromagnetic, piezoelectric or magnetostrictive actuators. Obviously, the unidirectionality of the generated flow, depending of the orientation of the punched loops, is a limitation for applications, in which a momentum transfer in both directions is needed, e.g. for locomotion of swimming objects. On the other hand, there are several potential use cases, for which bidirectionality is not necessary or even not wanted - for instance, when objects or gas bubbles in the liquid should be transported only into a given direction. Another advantage with respect to technical applications is the simplicity of the control of the movement of the individual ciliary elements, which is determined by the parameters of the exciting acoustic wave. The vibra- tional phase difference between neighbouring elements, for instance, which may influence the flow velocity in a similar way as in the metachronal wave of natural cilia array, depends only on their distance on the tape and the wavelength of the exciting wave and can easily be changed by the excitation frequency. Furthermore, via the amplitude of the excitation voltage the flow velocity can be controlled as well.

In addition it should be kept in mind that the properties of the punched tape used in this study and thus the operation parameters were given by the manufacturer, but certainly can be varied by future engineering. In particular the geometrical dimensions and arrangements of the punch holes and loops including the shapes of the edges, the mechanical properties, the materials used and the modes and frequencies of the acoustic excitation may become adopted to the requirements of the prospective applications. Further parameter studies may provide a better appraisal of the usefulness in that respect.

\section{Conclusions and outlook}

Flow propulsion was achieved by guided acoustic waves on a thin metallic tape with protruded loops neighbouring punch holes resembling to ciliary structures. With a punched hook-and-loop tape streaming velocities of up to $60 \mathrm{~mm} \cdot \mathrm{s}^{-1}$ were achieved in water with remote vibrational excitation by a voice coil at a frequency of $185 \mathrm{~Hz}$. The streaming direction was determined by the orientation of the loops. The joints of the loops following the elliptical trajectories of the tape surface were identified as the principal agitators of the flow. Streaming was suppressed completely when the punch holes were closed with tape or the viscosity was increased by adding glycerol. The observed phenomena were explained by acoustic streaming near vibrating sharp edges, which occurs when the curvature radius of the edge is much smaller than the width of the viscous boundary layer. This condition was fulfilled for the joints of the loops, the chosen frequencies and the viscosity of water; numerical simulations also corroborated this interpretation. The realization of this pumping concept, which to some extent has been inspired by biological archetypes, is possible with commercially available parts and promises several applications: The 
modification of the streaming profile of a liquid near the wall of a conduit, the increase of mixing efficiency, the acceleration of electrochemical reactions at electrodes, the removal of gas bubbles or the enhancement of heat transfer across a liquid-solid interface by forced convection.

\section{Acknowledgment}

This research was supported by the European Fund of Regional Development (EFRE) within the project "InnoTerm" and from "Technologieallianz Oberfranken (TAO)". Valuable comments from Sabrina Tietze are gratefully acknowledged. Keyence Deutschland $\mathrm{GmbH}$ helped with the measurements of the curvature of the loop edges. Additional technical information about the properties of the hook-and-loop tape was provided by Hölzel Stanz- und Feinwerktechnik GmbH \& Co. KG.

Funding Open Access funding enabled and organized by Projekt DEAL.

Open Access This article is licensed under a Creative Commons Attribution 4.0 International License, which permits use, sharing, adaptation, distribution and reproduction in any medium or format, as long as you give appropriate credit to the original author(s) and the source, provide a link to the Creative Commons licence, and indicate if changes were made.

The images or other third party material in this article are included in the article's Creative Commons licence, unless indicated otherwise in a credit line to the material. If material is not included in the article's Creative Commons licence and your intended use is not permitted by statutory regulation or exceeds the permitted use, you will need to obtain permission directly from the copyright holder.

To view a copy of this licence, visit http://creativecommons.org/licenses/by/4.0/.

* All supplementary materials are available at https://doi.org/10.1007/s42235-021-0051-x.

\section{References}

[1] Squires T M, Quake S R. Microfluidics: Fluid physics at the nanoliter scale. Review of Modern Physics, 2005, 77, 977-1026.

[2] Whitesides G M. The origins and future of microfluidics.
Nature, 2006, 442, 368-373.

[3] Liu C B, Wang Y J, Ren L Q, Ren L. A review of biological fluid power systems and their potential bionic applications. Journal of Bionic Engineering, 2019, 16, 367-399.

[4] Sleigh M A. Cilia and Flagella, Academic Press, London, UK, 1974.

[5] Ibanez-Tallon I, Heintz N, Omran H. To beat or not to beat: Roles of cilia in development and disease. Human Molecular Genetics, 2003, 12, R27-R35.

[6] Van Der Schans C P. Bronchial mucus transport. Respiratory Care, 2007, 52, 1150-1156.

[7] Brokaw C J. Flagellar movement: A sliding filament model. Science, 1972, 178, 455-462.

[8] Osterman N, Vilfan A. Finding the ciliary beating pattern with optimal efficiency. Proceedings of the National Academy of Sciences of the United States of America, 2011, 108, 15727-15732.

[9] Geyer V F. Characterization of the Flagellar Beat of the Single Cell Green Alga Chlamydomonas Reinhardtii, Doctoral Thesis, Technical University Dresden, Dresden, Germany, 2013.

[10] Ma R, Klindt G S, Riedel-Kruse I H, Jülicher F, Friedrich B M. Active phase and amplitude fluctuations of flagellar beating. Physical Review Letters, 2014, 113, 048101.

[11] Satir P, Christensen S T. Structure and function of mammalian cilia. Histochemical Cell Biology, 2008, 129, 687-693.

[12] Sleigh M A. Metachronal coordination of the comb plates of the ctenophore pleurobrachia. Journal of Experimental Biology, 1968, 48, 111-125.

[13] Brennen C, Winet H. Fluid mechanics of propulsion by cilia and flagella. Annual Review Fluid Mechanics, 1977, 9, 339-398.

[14] Hussong J, Breugem W P, Westerweel J. A continuum model for flow induced by metachronal coordination between beating cilia. Journal of Fluid Mechanics, 2011, 684, $137-162$.

[15] Elgeti J, Gompper G. Emergence of metachronal waves in cilia arrays. Proceedings of the National Academy of Sciences of the United States of America, 2013, 110, 4470-4475.

[16] Den Toonder J M J, Onck P R. Artificial Cilia, Royal Society of Chemistry Publishing, Cambridge, UK, 2013.

[17] Den Toonder J M J, Onck P R. Microfluidic manipulation with artificial/bioinspired cilia. Trends in Biotechnology, 2013, 31, 85-91.

[18] Mayne R, Den Toonder J M J. Atlas of Cilia Bioengineering and Biocomputing, River Publishers, Wharton, USA, 2018. 
[19] Den Toonder J M J, Bos F, Broer D, Filippini L, Gillies M, De Goede J, Mol T, Reijme M, Talen W, Wilderbeek H, Khatavkar V, Anderson P. Artificial cilia for active micro-fluidic mixing. Lab on a Chip, 2008, 8, 533-541.

[20] Baltussen M, Anderson P, Bos F, Den Toonder J M J. Inertial flow effects in a micro-mixer based on artificial cilia. Lab on a Chip, 2009, 9, 2326-2331.

[21] Sareh S, Rossiter J, Conn A, Drescher K, Goldstein R. Swimming like algae: Biomimetic soft artificial cilia. Journal of the Royal Society, Interface, 2013, 10, 20120666.

[22] Dreyfus R, Baudry J, Roper M L, Fermigier M, Stone H A, Bibette J. Microscopic artificial swimmers. Nature, 2005, 437, 862-865.

[23] Evans B A, Shields A R, Lloyd Carroll R, Washburn S, Falvo M R, Superfine R. Magnetically actuated nanorod arrays as biomimetic cilia. Nano Letters, 2007, 7, 1428-1434.

[24] Khaderi S N, Craus C B, Hussong J, Schorr N, Belardi J, Westerweel J, Prucker O, Rühe J, Den Toonder J M J, Onck P R. Magnetically-actuated artificial cilia for microfluidic propulsion. Lab on a Chip, 2011, 11, 2002-2010.

[25] Fahrni F, Prins M W J, van Ijzendoorn L J. Micro-fluidic actuation using magnetic artificial cilia. Lab on a Chip, 2009, 9, 3413-3421.

[26] Shields A R, Fiser B L, Evans B A, Falvo M R, Washburn S, Superfine R. Biomimetic cilia arrays generate simultaneous pumping and mixing regimes. Proceedings of the National Academy of Sciences of the United States of America, 2010, 107, 15670-15675.

[27] Vilfan M, Potocnik A, Kavcic B, Osterman N, Poberaj I, Vilfan A, Babic D. Self-assembled artificial cilia. Proceedings of the National Academy of Sciences of the United States of America, 2010, 107, 1844-1847.

[28] Hussong J, Schorr N, Belardi J, Prucker O, Rühe J, Westerweel J. Experimental investigation of the flow induced by artificial cilia. Lab on a Chip, 2011, 11, 2017-2022.

[29] Kokot G, Vilfan M, Osterman N, Vilfan A, Kavcic B, Poberaj I, Babic D. Measurement of fluid flow generated by artificial cilia. Biomicrofluidics, 2011, 5, 034103.

[30] Khaderi S, Hussong J, Westerweel J, den Toonder J M J, Onck P R. Fluid propulsion using magnetically-actuated artificial cilia - Experiments and simulations. RSC Advances, 2013, 3, 12735-12742.

[31] Wang Y, Gao Y, Wyss H M, Anderson P D, den Toonder J M J. Artificial cilia fabricated using magnetic fiber drawing generate substantial fluid flow. Microfluidics and Nanoflu- idics, 2015, 18, 167-174.

[32] Chen C Y, Cheng L Y, Hsu C C, Mani K. Microscale flow propulsion through bioinspired and magnetically actuated artificial cilia. Biomicrofluidics, 2015, 9, 034105.

[33] Wu Y A, Panigrahi B, Lu Y H, Chen C Y. An integrated artificial cilia based microfluidic device for micropumping and micromixing applications. Micromachines, 2017, 8, 260.

[34] Zhang S Z, Wang Y, Lavrijsen R, Onck P R, den Toonder J M J. Versatile microfluidic flow generated by moulded magnetic artificial cilia. Sensors and Actuators B: Chemical, 2018, B263, 614-624.

[35] Hanasoge S, Hesketh P J, Alexeev A. Microfluidic pumping using artificial magnetic cilia. Microsystems \& Nanoengineering, 2018, 4, 11.

[36] van Oosten C L, Bastiaansen C W M, Broer D J. Printed artificial cilia from liquid-crystal network actuators modularly driven by light. Nature Materials, 2009, 8, 677-682.

[37] Gorissen B, de Volder M, Reynaerts D. Pneumatically-actuated artificial cilia array for biomimetic fluid propulsion. Lab on a Chip, 2015, 15, 4348-4355.

[38] Brücker C, Keissner A. Streaming and mixing induced by a bundle of ciliary vibrating micro-pillars. Experiments in Fluids, 2010, 49, 57-65.

[39] Oh K, Smith B, Devasia S, Riley J J, Chung J H. Characterization of mixing performance for bio-mimetic silicone cilia. Microfluidics \& Nanofluidics, 2010, 9, 645-655.

[40] Kongthon J, Devasia S. Feedforward control of piezoactuator for evaluating cilia-based micro-mixing. Proceedings 18th IFAC World Congress, Milano, Italy, 2011, 12727-12732.

[41] Keißner A, Brücker C. Directional fluid transport along artificial ciliary surfaces with base-layer actuation of counter-rotating orbital beating patterns. Soft Matter, 2012, 8, 5342-5349.

[42] Brücker C, Schnakenberg U, Rockenbach A, Mikulich V. Effect of cilia orientation in metachronal transport of microparticles. World Journal of Mechanics, 2017, 7, 73453.

[43] Orbay S, Ozcelik A, Bachman H, Huang T J. Acoustic actuation of in situ fabricated artificial cilia. Journal of Micromechanics and Microengineering, 2018, 28, 025012.

[44] Zhou Z G, Liu Z W. Biomimetic cilia based on MEMS technology. Journal of Bionic Engineering, 2008, 5, 358-365.

[45] Suh J W, Darling R B, Böhringer K F, Donald B R, Baltes H, Kovacs G T A. CMOS integrated ciliary actuator array as a general-purpose micromanipulation tool for small objects. 
Journal of Microelectromechanical Systems, 1999, 8, 483-496.

[46] Whiting J G H, Mayne R, Adamatzky A. A parallel modular biomimetic cilia sorting platform. Biomimetics, 2018, 3, 5.

[47] Whiting J G H, Mayne R, Melhuish C, Adamatzky A. A cilia-inspired closed loop sensor-actuator array. Journal of Bionic Engineering, 2018, 15, 526-532.

[48] Zhu J, Jiang X M, Zhong J, Duan Y Y. Polymer brushes and their possible applications in artificial cilia research. Molecular Medicine Reports, 2017, 15, 3936-3942.

[49] Rockenbach A, Mikulich V, Brücker C, Schnakenberg U. Fluid transport via pneumatically actuated waves on a ciliated wall. Journal of Micromechanics and Microengineering, 2015, 25, 125009.

[50] Rose J L. Ultrasonic Guided Waves in Solid Media, Cambridge University Press, New York, USA, 2014.

[51] Friend J, Yeo L Y. Microscale acoustofluidics: Microfluidics driven via acoustics and ultrasonics. Review of Modern Physics, 2011, 83, 647-704.

[52] Iverson B D, Garimella S V. Recent advances in microscale pumping technologies: A review and evaluation. Microfluidics and Nanofluidics, 2008, 5, 145-174.

[53] Liang W, Lindner G. Investigation of droplet movement excited by Lamb waves on a non-piezoelectric substrate. Journal of Applied Physics, 2013, 114, 044501.

[54] Lindner G, Friedrich D. Apparatus for Producing and/or Detecting a Flow in a Medium, European Patent Office, 2011, EP 2545369 B1.

[55] Cegla F B, Cawley P, Lowe M J S. Material property measurement using the quasi-Scholte mode - A waveguide sensor. The Journal of the Acoustical Society of America, 2005, 117, 1098-1107.

[56] Yu L Y, Tian Z H. Case study of guided wave propagation in a one-side water-immersed steel plate. Case Studies in Nondestructive Testing and Evaluation, 2015, 3, 1-8.

[57] Tietze S, Lindner G. Visualization of the interaction of guided acoustic waves with water by light refractive vi- brometry. Ultrasonics, 2019, 99, 105955.

[58] Ovchinnikov M, Zhou J, Yalamanchili S. Acoustic streaming of a sharp edge. The Journal of the Acoustic Society of America, 2014, 136, 22-29.

[59] Giurgiutiu V. Structural Health Monitoring with Piezoelectric Wafer Active Sensors, 2nd ed, Academic Press, Oxford, UK, 2014.

[60] Andreassen E, Manktelow K, Ruzzene M. Directional bending wave propagation in periodically perforated plates. Journal of Sound \& Vibration, 2015, 335, 187-203.

[61] Techet A H, Hover F S, Triantafyllou M S. Vortical patterns behind a tapered cylinder oscillating transversely to a uniform flow. Journal of Fluid Mechanics, 1998, 363, 79-96.

[62] Gauger E M, Downton M T, Stark H. Fluid transport at low Reynolds number with magnetically actuated artificial cilia. The European Physical Journal E, 2009, 28, 231-242.

[63] Geyer V F, Jülicher F, Howard J, Friedrich B M. Cell-body rocking is a dominant mechanism for flagellar synchronization in a swimming alga. Proceedings of the National Academy of Sciences of the United States of America, 2013, 110, 18058-18063.

[64] Huang P H, Xie Y L, Ahmed D, Rufo J, Nama N, Chen Y C, Chan C Y, Huang T J. An acoustofluidic micromixer based on oscillating sidewall sharp-edges. Lab on a Chip, 2013, 13, 3847-3852.

[65] Nama N, Huang P H, Huang T Y, Costanzo F. Investigation of acoustic streaming patterns around oscillating sharp edges. Lab on A Chip, 2014, 14, 3824-2836.

[66] Nama N, Huang P H, Huang T Y, Costanzo F. Investigation of micromixing by acoustically oscillated sharp-edges. Biomicrofluidics, 2016, 10, 024124.

[67] Mohanty S, Siciliani de Cumis U, Solsona M, Misra S. Bi-directional transportation of micro-agents induced by symmetry-broken acoustic streaming. AIP Advances, 2019, 9, 035352.

[68] Jana S, Um S H, Jung S. Paramecium swimming in capillary tube. Physics of Fluids, 2012, 24, 041901. 